\title{
HLA-A2-Restricted Synthetic Glioma Antigen Peptides Vaccine
}

National Cancer Institute

\section{Source}

National Cancer Institute. HLA-A2-Restricted Synthetic Glioma Antigen Peptides Vaccine. NCl Thesaurus. Code C82661.

A synthetic peptide cancer vaccine consisting of HLA-A2-restricted peptides derived from glioma-associated antigens (GAA) with potential immunostimulating and antineoplastic activities. Upon administration, HLA-A2-restricted synthetic glioma antigen peptides vaccine may stimulate the host immune system to mount a cytotoxic T lymphocyte $(\mathrm{CTL})$ response ag ainst tumor cells expressing the corresponding GAAs, resulting in glioma tumor cell lysis. HLA-A2 is an MHC class I molecule that presents antigenic peptides to CD8+ T cells; epitope design restricted to epitopes that bind most efficiently to HLA-A2 may improve antigenic peptide immunogenicity. 